\title{
Multifractal analysis of radar rainfall fields over the area of Rome
}

\author{
G. Calenda ${ }^{1}$, E. Gorgucci ${ }^{2}$, F. Napolitano ${ }^{3}$, A. Novella ${ }^{1}$, and E. Volpi ${ }^{1}$ \\ ${ }^{1}$ Department of "Scienze dell'Ingegneria Civile", University of "Roma Tre", Rome, Italy \\ ${ }^{2}$ Institute of Atmospheric Sciences and Climate, National Research Council, Rome, Italy \\ ${ }^{3}$ D.I.T.S. no. 37, University of "La Sapienza”, Rome, Italy
}

Received: 24 October 2004 - Revised: 8 July 2005 - Accepted: 13 July 2005 - Published: 17 August 2005

\begin{abstract}
A scale-invariance analysis of space and time rainfall events monitored by meteorological radar over the area of Rome (Italy) is proposed. The study of the scale-invariance properties of intense precipitation storms, particularly important in flood forecast and risk mitigation, allows to transfer rainfall information from the large scale predictive meteorological models to the small scale hydrological rainfall-runoff models.

Precipitation events are monitored using data collected by the polarimetric Doppler radar Polar 55C (ISAC-CNR), located $15 \mathrm{~km}$ Southeast from downtown. The meteorological radar provides the estimates of rainfall intensity over an area of about $10000 \mathrm{~km}^{2}$ at a resolution of $2 \times 2 \mathrm{~km}^{2}$ in space and 5 min in time.

Many precipitation events have been observed from autumn 2001 up to now. A scale-invariance analysis is performed on some of these events with the aim at exploring the multifractal properties and at understanding their dependence on the meteorological large-scale conditions.
\end{abstract}

\section{Introduction}

The identification of space-time scaling behaviour of rainfall fields is fundamental for the development and the calibration of multifractal disaggregation models. These models allow to transfer rainfall information from the large scale predictive meteorological models to the smaller scale hydrological rainfall-runoff models.

The analysis of observed storms shows that the estimated parameters of downscaling models change from storm to storm, depending on the climatic situation and on the large scale meteorological conditions (Over and Gupta, 1994; Olsson and Niemczynowicz, 1996; Perica and FoufoulaGeorgiou, 1996; Svensson et al., 1996; Deidda, 2000; Deidda et al., 2004). Moreover, to reproduce the statistical prop-

Correspondence to: E. Volpi

(evolpi@uniroma3.it) erties jointly observed in space and time, the space and time scales must be related to the forcing factors of the events (Lovejoy and Schertzer; 1987; Venugopal et al., 1999; Deidda, 2000; Deidda et al., 2004).

This paper deals with the analysis of the space and time scaling properties of the rainfall events observed over the metropolitan area of Rome, in order to calibrate the parameters of an efficient disaggregation model for intense rainfall events.

In order to investigate the dependence of the model parameters on the characteristics of the storms, different meteorological conditions forcing intense precipitation events over the area of Rome have to be analysed. High resolution rainfall monitoring both in space and time over the area of Rome is possible thanks to the recent installation (2001) of the weather radar Polar 55C, managed by the Institute of Atmospheric Sciences and Climate of the National Research Council (ISAC-CNR). Some preliminary results are presented in this paper, due to the limited number of intense rainfall events registered until now. The monitoring of intense rainfall events over the study area is currently ongoing.

Some theoretical aspects of the multifractal properties of space-time rainfall fields are introduced in Sect. 2, where the multifractal model used for rainfall synthetic generation is described; the acquisition and processing of the available radar data are described in Sect. 3; the performed multifractal analysis is discussed in Sect. 4 and, finally, some conclusions are drawn in Sect. 5.

\section{Multifractal properties of space-time rainfall fields}

The $\lambda$-scale measure of the positive random field $P(\boldsymbol{x})$, $\boldsymbol{x} \in[0, L]^{n}$,

$$
\mu_{x_{1}, x_{2}, \ldots x_{n}}(\lambda)=\int_{x_{1}}^{x_{1}+\lambda} \int_{x_{2}}^{x_{2}+\lambda} \ldots \int_{x_{n}}^{x_{n}+\lambda} P\left(\xi_{1}, \xi_{2}, \ldots \xi_{n},\right) d \xi_{1} d \xi_{2} . . d \xi_{n}
$$


Table 1. Radar data set used to perform multifractal analyses.

\begin{tabular}{ll}
\hline Date & Temporal interval \\
\hline October 2003 & $22 / 10 / 2003$ 6:30-23/10/2003 16:40 \\
November 2003 & 26/11/2003 7:10-27/11/2003 3:50 \\
February 2004 & 26/02/2004 11:52-27/02/2004 5:52 \\
March 2004 & $01 / 03 / 20048: 10-15: 20$
\end{tabular}

is multifractal if the $q$-moments of the measure satisfy the multiple scaling condition:

$S_{q}(\lambda)=\left\langle\left[\mu_{x}(\lambda)\right]^{q}\right\rangle \sim \lambda^{\varsigma(q)}$

where $\langle\cdot\rangle$ is the ensemble and spatial average at the $\lambda$ scale. The exponents $\zeta(q)$ are called multifractal if they are non-linear functions of $q$.

In order to make the time scale $\tau$ dimensionally homogeneous with the spatial scales $\lambda$ it can be rescaled by a velocity parameter $U$, so that $\lambda=\tau U$.

The velocity parameter $U$ depends on whether the rainfall field can be regarded as a self-similar (space-time isotropy) or a self-affine (space-time anisotropy) scale invariant process. In the first case $U$ is constant with the scale, while in the second case it changes according to the power law $U_{\lambda} \sim \lambda^{H}$. Following the generalised scale invariance notation (Lovejoy and Schertzer, 1987) $H$ is called scaling anisotropy exponent.

An estimate of the velocity parameter $U$ can be obtained through the equivalence of the auto-correlation functions evaluated along the spatial axis, $x$ and $y$, and the rescaled temporal axis $t \rho_{x}(\lambda)=\rho_{y}(\lambda)=\rho_{t}(\lambda / U)$ (Deidda, 2000).

\subsection{Simulation of synthetic rainfall fields}

The multifractal cascade model STRAIN (Deidda, 2000) is used to generate synthetic space-time rainfall fields. Synthetic fields are obtained as a wavelet expansion with coefficients extracted from a stochastic cascade. The STRAIN model is based on the log-Poisson generator $\eta e^{A} \beta^{y}$, where $y$ is a Poisson distributed random variable with mean $c$. A conservation condition on the mean of the simulated field gives $A=c(1-\beta)$.

The expected multifractal exponents $\zeta(q)$ depend on the dimension $n$ of the region supporting the measure $\mu$, on the branching number of the random cascade, assumed dyadic, and on the statistics of the generator $\eta$ :

$\varsigma(q)=n q+\frac{c}{\ln 2}\left[q(\beta-1)-\left(\beta^{q}-1\right)\right]$

with $n=3$ for space-time rainfall fields.

The estimates of the model parameters $c$ and $\beta$ can be obtained minimising the following squared difference function:

$S(c, \beta)=\sum_{q}\left[\frac{\varsigma^{\prime}(q)-\varsigma(q)}{q-1}\right]^{2}$ where the $\zeta^{\prime}(q)$ are the sample multifractal exponent, estimated by Eq. (2), the $\zeta(q)$ are their theoretical expectations, expressed by Eq. (3), and $(q-1)$ is a weight accounting for the estimation error of the $\zeta(q)$.

\section{Rainfall monitoring over the area of Rome}

The Polar $55 \mathrm{C}$ is a C-band $(5.5 \mathrm{GHz}, \lambda=5.4 \mathrm{~cm})$ Doppler dual polarized coherent weather radar with polarization agility and with a $0.9^{\circ}$ beam-width. The radar is located Southeast area of Rome $\left(41^{\circ} 50^{\prime} 24^{\prime \prime}\right.$ North, $12^{\circ} 38^{\prime} 50^{\prime \prime}$ East, $102 \mathrm{~m}$ a.s.l.). The radar reflectivity measures $Z$ cover a circular area with a radius of about $120 \mathrm{~km}$.

Many rainfall events were monitored from September 2001 until October 200, but only the most intense (Table 1) were selected to perform the multifractal analysis. Reflectivity measures were collected with a time resolution of about 5 min, over a single plan position indicator (PPI, $360^{\circ}$ in azimuth) at about $1.8^{\circ}$ of elevation (small deviations from this value may be due to strong winds).

Some preliminary analyses on PPI at different elevations were performed in order to find the best antenna elevation for rainfall estimation (Gorgucci et al., 1995; Russo et al., 2001). Low elevation angles are required to avoid contamination of the radar echoes by the melting layer; but lowelevation radar scans suffer from ground clutter contamination and blockage of radar beam by elevated ground targets or mountains. While ground clutter contamination can be removed using a filter, no processing procedure can recover the blocked echoes.

At the elevation of about $1.8^{\circ}$ some clutter was recognised (Fig. 1a). The visibility is reduced in the Southeast direction (azimuth $154^{\circ}$ and range between $5-15 \mathrm{~km}$ ), by the "Colli Albani" hills, with the highest peak on "Monte Cavo" (949 m a.s.1.), and in the East direction (azimuth $70-90^{\circ}$ and range $20 \mathrm{~km}$ ), by the "Monti Prenestini" ridge.

To remove the ground clutter, a filter based on the standard deviation of the measured differential reflectivity $\left(Z_{d r}\right)$ was used. $Z_{d r}$ is defined as the difference between reflectivity factors measured at the horizontal and at the vertical polarization; its standard deviation $\sigma\left(Z_{d r}\right)$ strongly increases with the orographic gradient. In the cells affected by ground clutter, where $\sigma\left(Z_{d r}\right)$ is not included in the range 0.09-0.5, the reflectivity has been reconstructed by linear interpolation on the maximum distance of $9 \mathrm{~km}$ (Fig. 1b). Since the beam is completely blocked by "Monte Cavo", no radar echo is received from the targets in the southeast quarter, where the radar map was completely removed (Fig. 1c). The potential contamination from hail and ice regions was eliminated enforcing an upper limit of $55 \mathrm{dBZ}$ for the reflectivity factor (Aydin et al., 1986).

The radar data were converted to rainfall rates (Fig. 1d) using an algorithm based on a reflectivity-rainfall relationship found by simulation for C-band:

$R_{Z h}=7.27 \cdot 10^{-2} Z_{h}^{0.62}$ 

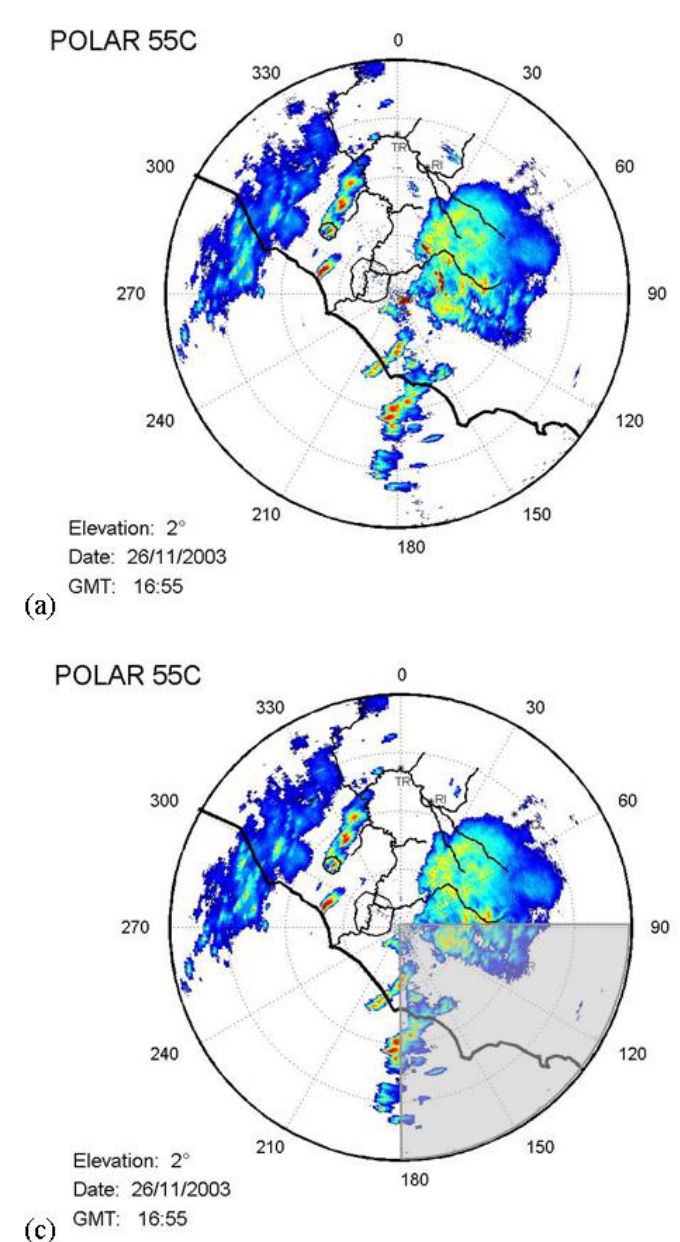
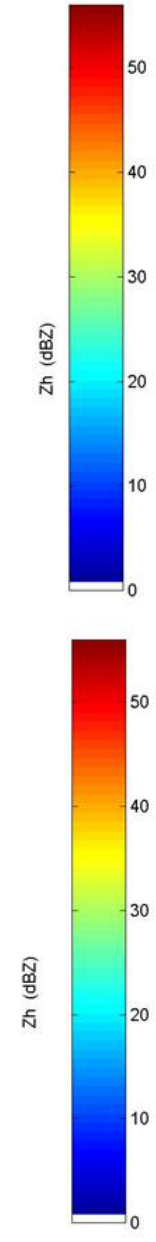

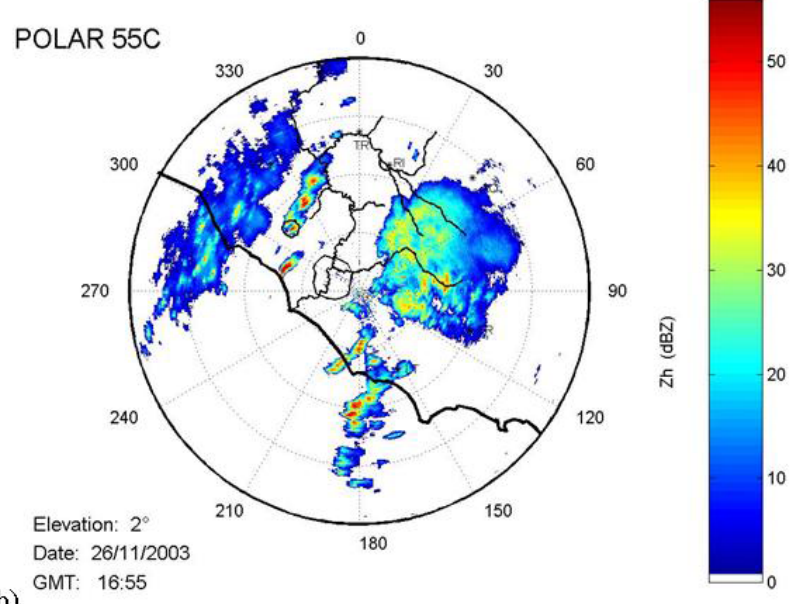

(b)

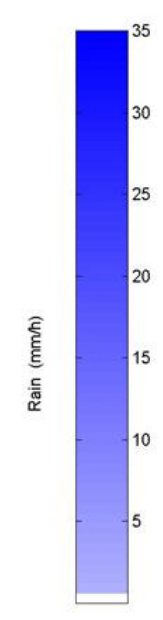

Fig. 1. Processing of a radar reflectivity map (26/11/2003, 16:55): (a) original map; (b) reflectivity map reconstructed by linear interpolation after ground clutter filtering; (c) reflectivity map without the region affected by beam blocking; d) rainfall intensity map.

where $Z$ is the reflectivity factor [dBZ] and $R_{Z h}$ is the rainfall intensity $\left[\mathrm{mm} \mathrm{h}^{-1}\right]$.

In order to investigate the scaling behaviour of Eq. (2) on a wide range of space and time scales, the rainfall field $R_{Z h}$ was interpolated on a rectangular grid with a $2 \times 2 \mathrm{~km}^{2}$ resolution over a domain size of $100 \times 100 \mathrm{~km}^{2}$ (Fig. 2).

\section{Multifractal properties of rainfall fields over the area of Rome}

Assuming the rainfall field as a self-similar process, the equivalence of the auto-correlation functions along the space and rescaled time dimensions was verified for two values of the velocity parameter $U=24 \mathrm{~km} \mathrm{~h}^{-1}$ and $U=48 \mathrm{~km} \mathrm{~h}^{-1}$, both values compatible with the discrete resolution of the radar fields in space $\left(\lambda_{0}=2 \mathrm{~km}\right)$ and time $\left(\tau_{0}=5 \mathrm{~min}\right)$.

In the case of $U=24 \mathrm{~km} \mathrm{~h}^{-1}$ the largest space scale of the investigated fields is $L=100 \mathrm{~km}$ and consequently the largest time scale is $T=L / U=250 \mathrm{~min}$. Six sequences of 50 consecutive radar scans were selected on the basis of the highest intensity level. The time interval and main statistics of the rainfall distribution (mean $I$, standard deviation $\sigma$ and variation coefficient $C V$ ) for each selected sequence are listed in Table 2. The event of March 2004 was too short to select such extended sequences. In the case of $U=48 \mathrm{~km} \mathrm{~h}^{-1}$ eighteen sequences were selected with the largest time scale $T=125 \mathrm{~min}$.

The average auto-correlation functions $\rho_{x}, \rho_{y}$ and $\rho_{t}$ obtained from all the selected sequences are plotted in Fig. 3. In the case of $U=24 \mathrm{~km} \mathrm{~h}^{-1}$ (Fig. 3a) the equivalence $\rho_{x}=\rho_{y}=\rho_{t}$ is not perfectly verified, but the relative distance between the three curves is small if compared to that from the curves obtained using the velocity parameter $U=48 \mathrm{~km} \mathrm{~h}^{-1}$ (Fig. 3b).

The multifractal analysis was performed for all the rainfall sequences (Table 2). The $q$-moments $S_{q}(\lambda)$ were evaluated for $\lambda$ scales ranging from $\lambda_{0}=2 \mathrm{~km}$ to $\lambda=32 \mathrm{~km}$, corresponding to the time scales in the range between $\tau_{0}=5 \mathrm{~min}$ and $\tau=80 \mathrm{~min}$, and for $q \in[0.2,6]$. The scaling law, described by Eq. (2), was verified with a correlation coefficient larger than 0.997 for all the selected sequences in the entire range of scales. Figure 4 shows the $\log$ - $\log$ plots of $S_{q}(\lambda)$ versus $\lambda$ for the first sequence. 


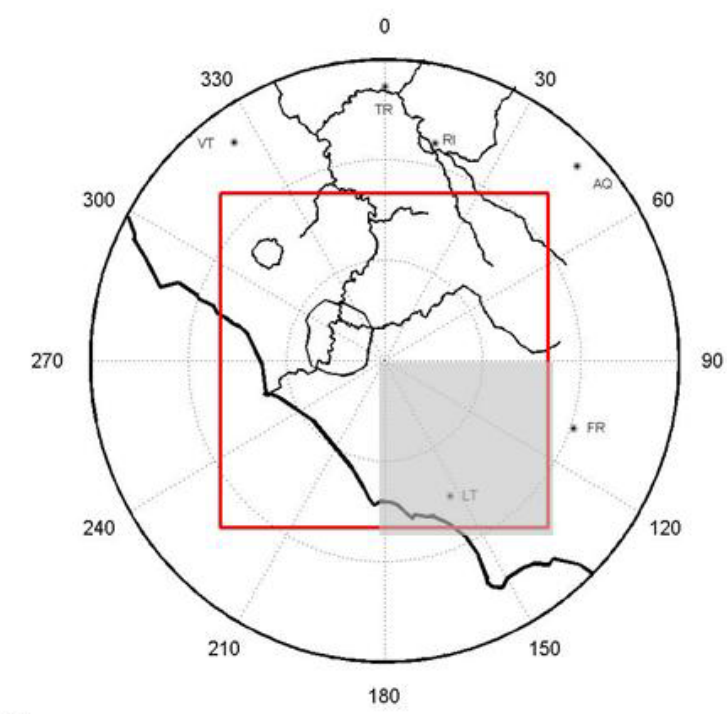

(a)

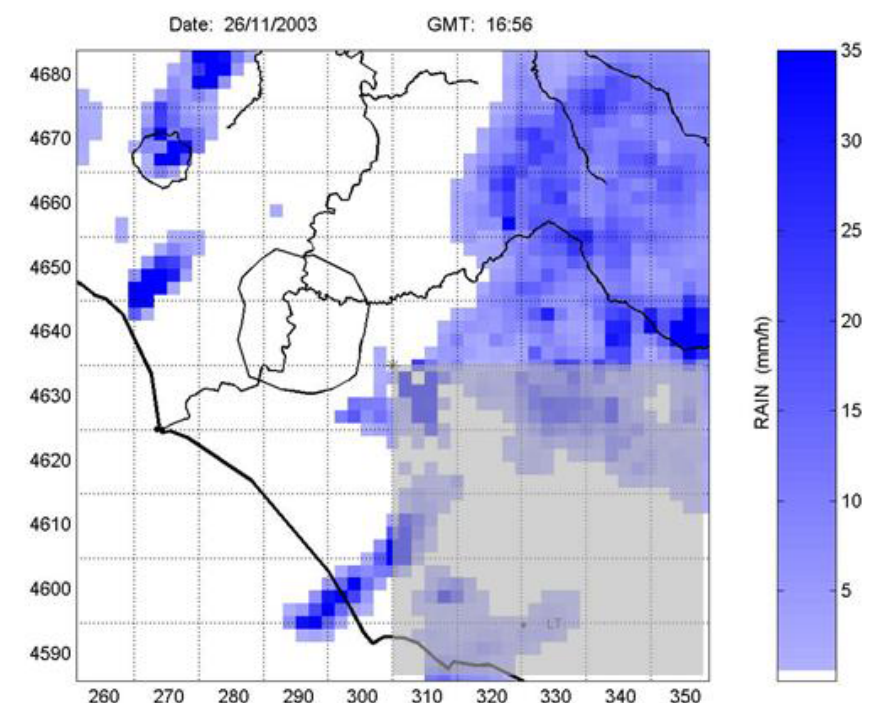

(b)

Fig. 2. (a) Rectangular grid included in the area monitored by the radar and (b) related interpolation of a rainfall intensity map (26/11/2003, $16: 55)$.

Table 2. Radar sequences selected assuming the velocity parameter $U=24 \mathrm{kmh}^{-1}$.

\begin{tabular}{clccc}
\hline & & & & \\
no. & Temporal interval & $I\left[\mathrm{~mm} \mathrm{~h}^{-1}\right]$ & $\sigma\left[\mathrm{mm} \mathrm{h}^{-1}\right]$ & $C V$ \\
\hline 1 & $22 / 10 / 200307: 01-22 / 10 / 200311: 06$ & 2.462 & 4.243 & 1.724 \\
2 & $22 / 10 / 200316: 50-22 / 10 / 200320: 55$ & 2.787 & 2.455 & 0.881 \\
3 & 26/11/2003 18:31-26/11/2003 22:36 & 1.264 & 3.292 & 2.604 \\
4 & 26/02/2004 12:52-26/02/2004 16:57 & 1.376 & 3.210 & 2.334 \\
5 & 26/02/2004 20:57-27/02/2004 01:02 & 0.326 & 1.629 & 4.996 \\
6 & 27/02/2004 01:07-27/02/2004 05:12 & 0.136 & 0.306 & 2.251 \\
& & & & \\
\hline
\end{tabular}

The sample multifractal exponents $\zeta^{\prime}(q)$ were estimated for each field. The sample exponents were used to estimate the $\log$-Poisson parameters $c$ and $\beta$ minimising the function in Eq. (4). In Fig. 5a to $f$ the sample exponents are compared to the theoretical values $\zeta(q)$ of the model (Eq. 3). The figures show that the selected sequences have a different behaviour, according to their variation coefficients: higher values of the variation coefficient imply also higher differences between the fractal (represented in Fig. 5 with the dashed line) and the estimated behaviours. Also the sequences extracted from the same event, as the sequences 4, 5 and 6, exhibit a different behaviour. As a consequence the estimated parameters exhibit a significant variation in the domain $0 \leq \beta \leq 1$ and $c \geq 0$.

The behaviour of the estimated parameters, considerably varying from sequence to sequence, was related to the storm characteristics. Following Deidda (2000) and Deidda et al. (2004), the relationships between the multifractal properties, represented by the model parameters $c$ and $\beta$, and the large-scale rain rate $I\left[\mathrm{~mm} \mathrm{~h}^{-1}\right]$ were investigated. Figure $6 \mathrm{a}$ presents the variability of the estimated values of $c$ and $\beta$ with $I$. The estimated parameters do not exhibit a dependence on the large-scale rain rate $I$. Figure 6 a shows that higher values of the $\beta$ parameter imply higher values of the $c$ parameter: in spite of the limited number of the analysed sequences, the corresponding estimated parameters $c$ and $\beta$ seem to exhibit a correlation that still has to be understood.

In order to get a better insight of the relationship between the multifractal properties and the mean value of the rainfall intensity $I$, considering that a experimental dependence exists between the parameters $c$ and $\beta$, a value $\beta=\beta_{1}$ common to all the sequences was fixed (Deidda, 2000) and a new estimate $c_{\beta}$ of the parameter $c$ was produced for each sequence.

As previously stated, $\beta$ shows a great variability in the range $(0,1)$, from $\beta=0.325$ for sequence 2 to $\beta=0.837$ for sequence 3. Because of this variability, the fixed value $\beta_{1}$ was chosen not as the mean estimated value of $\beta$, but as the value minimising the sum of the squared difference between the sample and the theoretical exponents for the global set of sequences. 

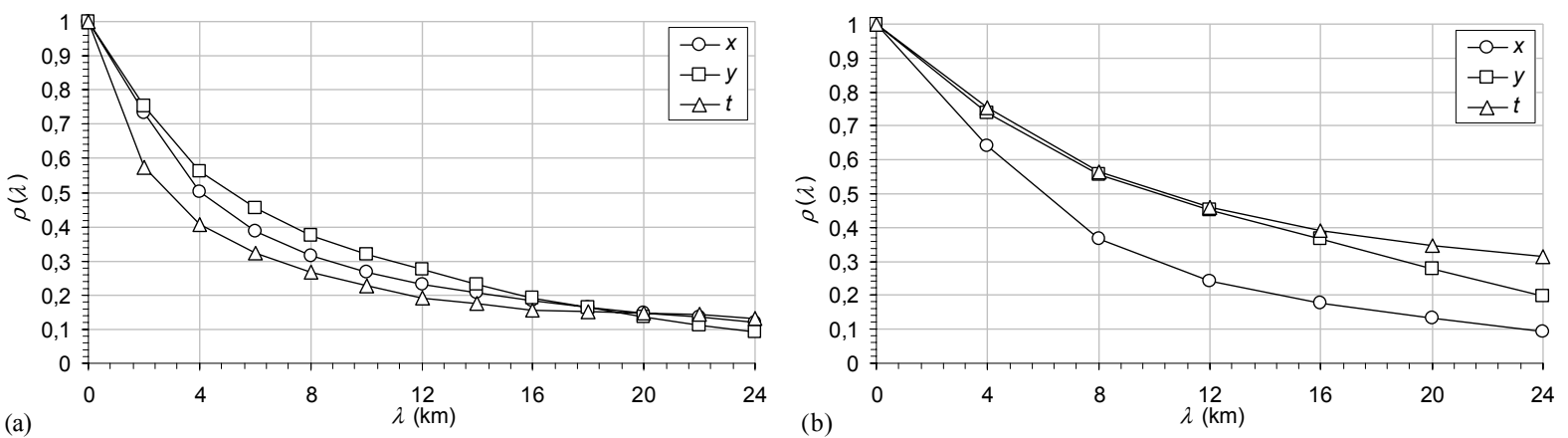

Fig. 3. Mean autocorrelation functions along the spatial directions, $x$ and $y$, and the rescaled temporal direction $U t$ evaluated assuming a velocity parameter (a) $U=24 \mathrm{~km} \mathrm{~h}^{-1}$ and (b) $U=48 \mathrm{~km} \mathrm{~h}^{-1}$.

The new values of the estimated parameters are listed in Table 3. The parameter $c_{\beta}$ shows a dependence on the mean intensity $I$, that, according to the literature results (Deidda et al., 2004), can be expressed by the exponential relation (Fig. 6b):

$c(I)=a e^{-\gamma I}+c_{\infty}$

with $a=2.217, \gamma=0.400$ and $c_{\infty}=0$. The exponential form of the relationship is fitting also if different values of the $\beta_{1}$ parameter are assumed, as shown in Fig. 7 where $\beta_{1}=e^{-1}$. The relationship (Eq. 6) is compared in Fig. 7 with the literature results obtained assuming the observed mean value $\beta=e^{-1}$.

The results don't show dependence of the parameter $\beta$ on the rain-rate $I$. The simplest assumption is to take $\beta$ constant with $I$, as indicated by previous literature results, although the estimates differ significantly from one sequence to the other. The hypothesis that the experimental variability of $\beta$ could be ascribed to a dependence on other characteristics of the storms or on seasonal or climatic factors cannot be ruled out. However, due to the small intensity and the limited number of the analysed events, is not possible to evaluate the dependence of $\beta$ on the meteorological conditions forcing the precipitation events.

\section{Summary and discussion}

The study aims at exploring the dependence of the estimated multifractal properties on the large scale meteorological conditions, large scale rain rate. The analysis was performed for rainfall events observed jointly in space and time.

A meteorological radar provides the rainfall estimates over the area of interest. Radar reflectivity measurements were collected and processed in order to obtain the rainfall intensity fields and perform a multifractal analysis in space and time.

The observed rainfall events were considered as selfsimilar processes, isotropic in space and time, assuming the velocity parameter $U=24 \mathrm{~km} \mathrm{~h}^{-1}$. Under this assumption, all the selected fields show a scaling behaviour in the range of the space scales from 2 to $32 \mathrm{~km}$, corresponding to the time

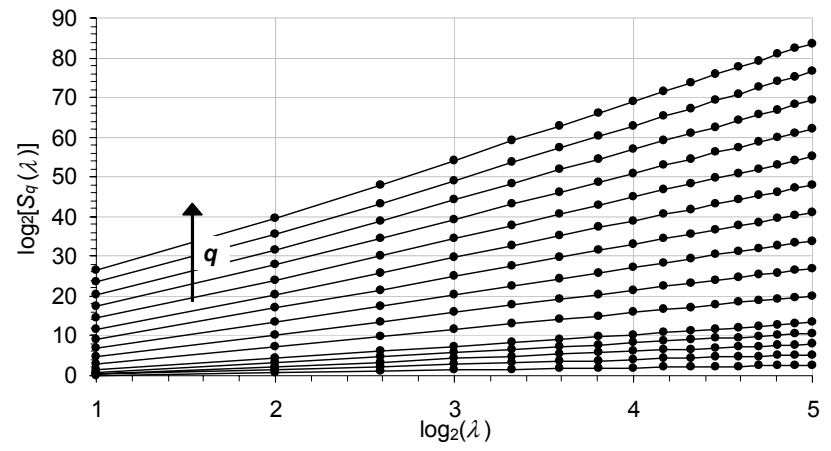

Fig. 4. Log-log plot of $S_{q}(\lambda)$ versus $\lambda$ for the first sequence (October 2003).

Table 3. Estimated log-Poisson parameters,

\begin{tabular}{ccccc}
\hline no. & $C$ & $\beta$ & $c_{\beta}$ & $\beta_{1}$ \\
\hline 1 & 1.594 & 0.586 & 1.120 & \\
2 & 3.400 & 0.837 & 0.446 & \\
3 & 0.918 & 0.325 & 1.439 & \\
4 & 2.081 & 0.656 & 1.060 & 0.489 \\
5 & 1.873 & 0.403 & 2.412 & \\
6 & 3.206 & 0.646 & 1.719 & \\
\hline
\end{tabular}

scales from 5 to $80 \mathrm{~min}$. The estimated multifractal properties for different fields present a significant variability in the domain $0 \leq \beta \leq 1$ and $c \geq 0$.

The STRAIN model, based on log-Poisson generator, was used to reproduce the observed properties. The estimated $\log$-Poisson parameters $c$ and $\beta$ exhibit a significant variation in the domain $0 \leq \beta \leq 1$ and $c \geq 0$. But the estimated parameters show a mutual dependence that still has to be understood. Fixing $\beta, c$ reveals a relationship with the large scale rain rate $I$, as suggested in some previous literature results. Due to the variability of the estimated $\beta$ parameters, an open question must be investigated about this parameter: whether the value of $\beta$ should be unique or should be influenced by other storm characteristics and by seasonal and climatic factors. 

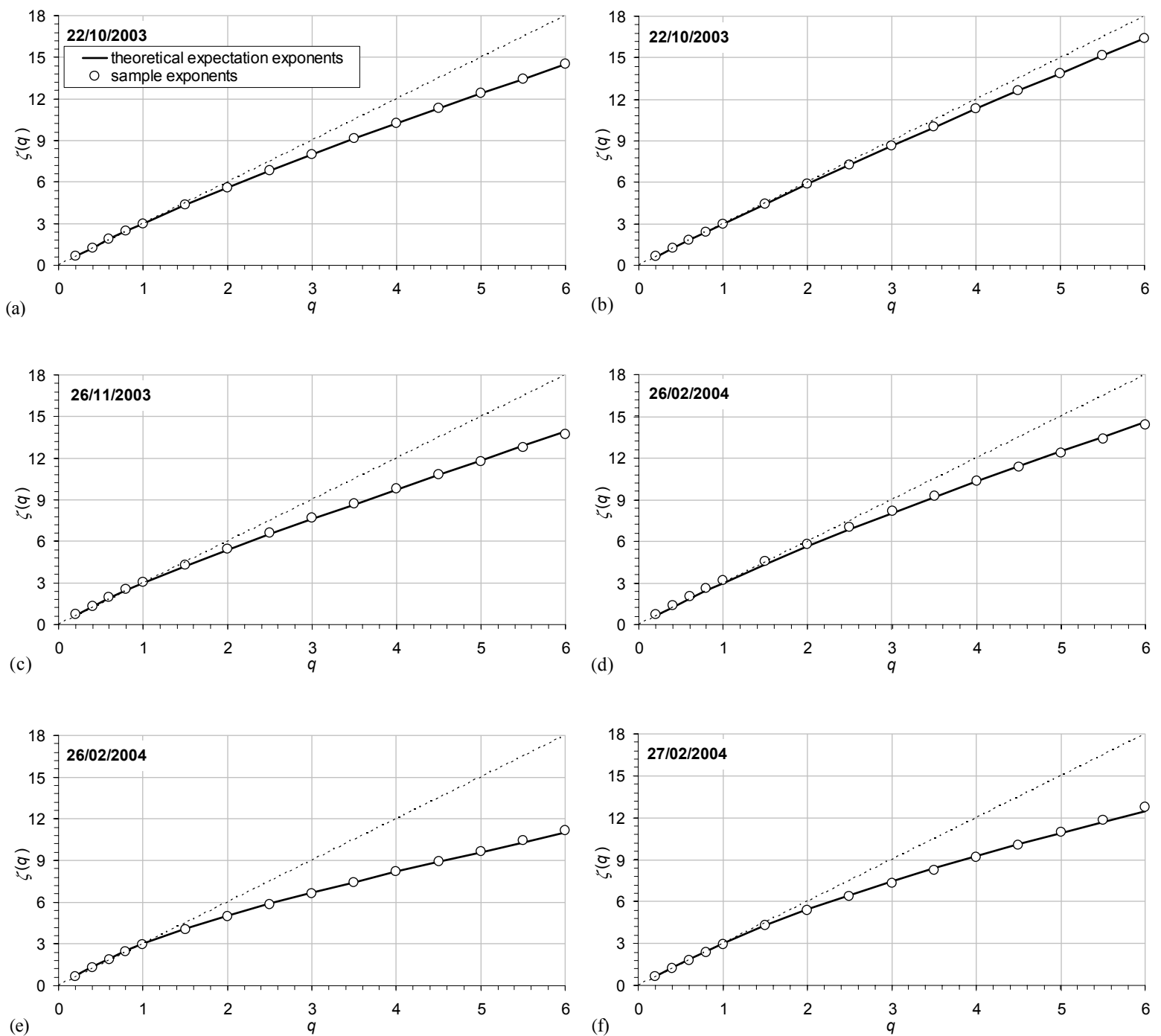

Fig. 5. Sample multifractal exponents $\zeta^{\prime}(q)$ for all the sequences compared to the theoretical multifractal exponents $\zeta(q)$ of the STRAIN model.

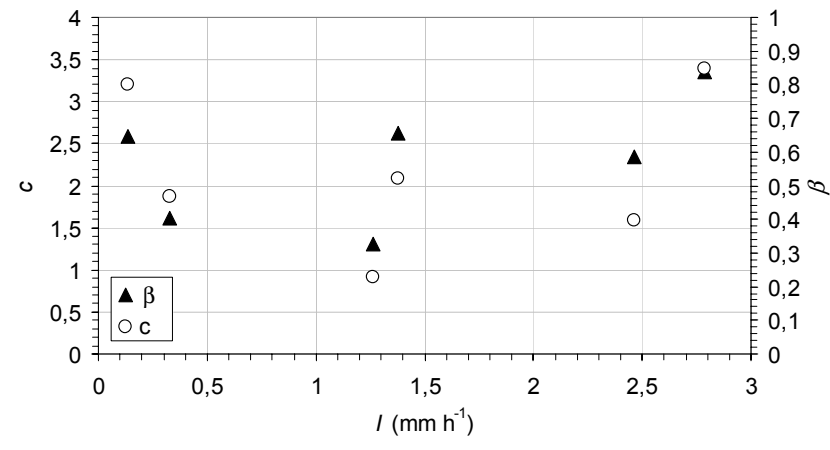

(a)

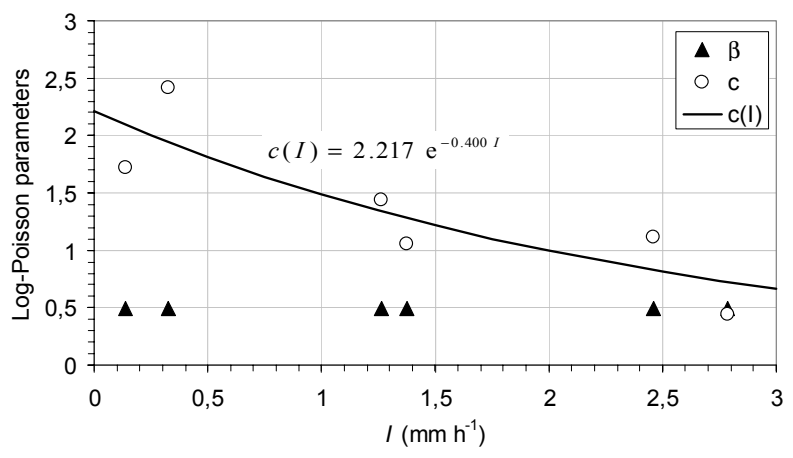

(b)

Fig. 6. STRAIN parameters versus the mean rainfall intensity $I$ : (a) $c$ and $\beta$; (b) $c_{\beta}$ and $\beta_{1}$. 


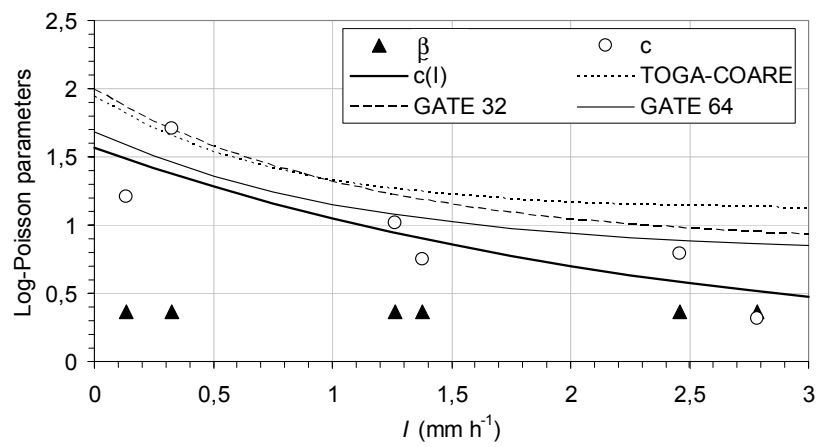

Fig. 7. STRAIN parameters versus the mean rainfall intensity $I$, estimated assuming $\beta=e^{-1}$. The estimated relationship $c(I)$ is compared with literature results (Deidda et al., 2004).

The obtained results are still preliminary due to the limited number of intense events as yet collected by the meteorological radar; but the preparation of a more extended data set of rainfall storms over the area of Rome is currently ongoing.

Edited by: L. Ferraris

Reviewed by: anonymous referees

\section{References}

Aydin, K. T., Seliga, T. A., and Balaji, V.: Remote sensing of hail with dual-linear polarization radar, Journal of Climate and Applied Meteorology, 25, 1475-1484, 1986.
Deidda, R.: Rainfall downscaling in a space-time multifractal framework, Water Resour. Res., 36(7), 1779-1794, 2000.

Deidda, R., Badas, M. G., and Piga, E.: Space-time scaling in high intensity Tropical Ocean Global Atmosphere Coupled OceanAtmosphere Response Experiment (TOGA-COARE) storms, Water Resour. Res., 40, doi:10.1029/2003WR002574, 2004.

Gorgucci, E., Scarchilli, G., and Chandrasekar, V.: Radar and surface measurements of rainfall during CaPE, Journal of Applied Meteorology, 34, 1570-1577, 1995.

Olsson, J. and Niemczynowicz, J.: Multifractal analysis of daily spatial rainfall distributions, J. Hydrol., 187, 29-43, 1996.

Over, T. M. and Gupta, V. K.: Statistical analysis of mesoscale rainfall: dependence of a random cascade generator on large-scale forcing, Journal of Applied Meteorology, 33, 1526-1542, 1994.

Perica, S. and Foufoula-Georgiou, E.: Linkage of scaling and thermodynamic parameters of rainfall: Results from midlatitude mesoscale convective systems, J. Geophys. Res., 10(D3), 74317448, 1996.

Russo, F., Gorgucci, E., Napolitano, F., and Ubertini, L.: Radar rainfall estimates and raingauge measurements comparison: the Sesia basin case study during the Mesoscale Alpine Program, Proceedings of the III EGS Plinius Conference, Baja Sardinia, Italy, Abstr. No. p3c0045, 2001.

Schertzer, D. and Lovejoy, S.: Physical modelling and analysis of rain and clouds by anisotropic scaling multiplicative processes, J. Geophys. Res., 97(D8), 9696-9714, 1987.

Svensson, C., Olsson, J., and Niemczynowicz, J.: Multifractal properties of daily rainfall in two different climates, Water Resour. Res., 32(8), 2463-2472, 1996.

Venugopal, V., Foufoula-Georgiou, E., and Sapozhnikov, V.: Evidence of dynamic scaling in space-time rainfall, J. Geophys. Res., 104(D24), 31 599-31 610, 1999. 